\title{
Characterization of the density and body size of a Gammarus pulex (Crustacea: Amphipoda) population in subsurface sediments reflects the sampling technique used
}

\author{
Rachel Stubbington $\mathbb{D} \cdot$ John-Paul Hogan $\cdot$ Paul J. Wood
}

Received: 14 July 2016/Revised: 1 October 2016/Accepted: 1 October 2016

(C) The Author(s) 2016. This article is published with open access at Springerlink.com

\begin{abstract}
The hyporheic zone and its invertebrate fauna play vital roles in the functioning of lotic ecosystems. However, although sampling invertebrates from subsurface sediments is recognized as challenging, few studies have quantified the effectiveness of common sampling techniques. We conducted laboratory experiments to compare two common, semi-quantitative pump-sampling techniques-Bou-Rouch and vacuumpump sampling. We determined the proportion of a Gammarus pulex (Crustacea: Amphipoda) population sampled by each method in five sediment treatments comprising coarse $(16-32 \mathrm{~mm})$, medium $(8-16 \mathrm{~mm})$, and fine (4-8 $\mathrm{mm}$ ) gravel and combinations thereof. We compared the body size of individuals sampled and not sampled by each technique to the population mean. Density estimates obtained using both methods were low: $33 \pm 5$ and $5 \pm 1 \%$ of the population present for Bou-Rouch and vacuum-pump samples, respectively. Density estimates were significantly higher for BouRouch than for vacuum-pump samples in four sediment
\end{abstract}

Handling editor: Koen Martens

R. Stubbington $(\bowtie) \cdot$ J.-P. Hogan

School of Science and Technology, Nottingham Trent University, Clifton Campus, Nottingham NG11 8NS, UK

e-mail: rachel.stubbington@ntu.ac.uk

\section{P. J. Wood}

Centre for Hydrological and Ecosystem Science, Department of Geography, Loughborough University, Loughborough, Leicestershire LE11 3TU, UK treatments, but were comparable in coarse gravel. The body size of organisms captured by the Bou-Rouch technique was comparable to the population mean, whereas those in vacuum-pump samples were smaller. With hyporheic invertebrates suggested as future biomonitors of ecosystem health, we recommend Bou-Rouch sampling as the more effective pumpsampling method for community characterization.

Keywords Hyporheos - Hyporheic refuge · Bioindicators $\cdot$ Sampling techniques $\cdot$ Sampling effectiveness $\cdot$ Mesocosm experiments

\section{Introduction}

The subsurface sediments of the hyporheic zone are inhabited by an abundant and diverse invertebrate fauna that includes macroinvertebrates and meiofauna, benthic taxa and obligate stygobites, and permanent and temporary inhabitants (Boulton, 2000). Temporary residents include predominantly benthic macroinvertebrate taxa, which may use the subsurface interstices as a refuge from both biotic interactions such as predation (McGrath et al., 2007), and to escape adverse abiotic conditions in the surface stream. For example, hyporheic refuge use by the predominantly benthic crustacean Gammarus pulex Linnaeus (Crustacea: Amphipoda) has been inferred from changes in its vertical distribution during both floods (Dole-Olivier et al., 1997) and low flows 
(Stubbington et al., 2011a, b), the latter refuge use being linked to increased biotic interactions in benthic sediments. In addition, Vander Vorste et al. (2016a) identified vertical migration from the hyporheic zone as the primary process promoting recovery of a benthic macroinvertebrate community after a drying event.

Research examining hyporheic invertebrate communities is increasing, including their provision of ecosystem services (Marshall \& Hall, 2004; Boulton et al., 2008)and their responses to environmental change (Jones et al., 2015; Stubbington et al., 2015). In addition, hyporheic invertebrates are potential biomonitors of ecosystem health, for example in groundwater-dominated ecosystems (Malard et al., 1996; Tomlinson et al., 2007), and in temporary streams, where invertebrates may persist in hyporheic sediments after benthic communities are altered by surface water loss (Leigh et al., 2013; Boulton, 2014). Evaluation of the potential role of hyporheic invertebrate communities in biomonitoring programmes may become an increasing priority for national regulatory agencies, due to legal drivers such as the EU Water Framework Directive (2000/60/EC; European Commission, 2000; Reyjol et al., 2014).

Despite this increasing interest, sampling invertebrates from the hyporheic zone remains challenging due to the inaccessibility of subsurface sediments (Palmer, 1993). Quantitative methods such as freezecoring, standpipe corers, and colonization chambers have been evaluated previously (e.g. Fraser \& Williams, 1997; ISO, 2015; and see Stubbington et al., 2016), and due to their significant limitations, many studies of hyporheic invertebrate communities have instead used semi-quantitative methods. In particular, semi-quantitative pump-sampling methods are widely used (e.g. Dole-Olivier et al., 1997; Stubbington et al., 2011a, b; 2015; Datry, 2012; Vander Vorste et al., 2016a), their logistic advantages including ease of operation, rapid sample collection, modest financial outlay, and minimal ongoing sampling costs. In addition, because pump sampling causes only limited, localized disturbance to the sediments, these methods allow immediate sample collection (rather than requiring prior installation of equipment) and, if pipes are left in situ, allow repeated sample collection from specific points. Limitations of these methods include a potential bias towards the collection of smaller organisms, which are less able to resist the suction forces exerted by pump sampling and which move more freely through interstices (Fraser \& Williams, 1997; Boulton et al., 2004a; Tanaka et al., 2014).

The main two hyporheic invertebrate pump-sampling methods are Bou-Rouch sampling (Bou \& Rouch, 1967) and vacuum-pump sampling (Boulton et al., 1992). These methods have been used to study regional biodiversity (Dole-Olivier et al., 2009); longitudinal (Malard et al., 2003) and sub-reach (Davy-Bowker et al., 2006) spatial variability in community composition; temporal changes in community composition in response to hydrological variability (Stubbington et al., 2011a); and the vertical distribution of fauna (Dole-Olivier \& Marmonier, 1992; Dole-Olivier et al., 1997). Stubbington et al. (2016) compared the two pump-sampling methods in contrasting rivers across two biogeographical regions and recorded higher abundance and richness estimates in Bou-Rouch samples. In addition, Dole-Olivier et al. (2014) collected Bou-Rouch samples within the confines of a quantitative benthic sampler and found that this method captured $14.5 \%$ of the organisms present. However, no controlled experiments have been conducted to determine or compare the capture rates of pump-sampling methods, limiting interpretation of data collected by studies using these techniques.

We conducted experiments in laboratory-based mesocosms to compare the invertebrate abundance estimates obtained by Bou-Rouch and vacuum-pump sampling methods. Our first hypothesis was that abundance estimates would be higher in Bou-Rouch samples than in vacuum-pump samples. To test this hypothesis, we used each technique to sample a $G$. pulex population of known size and then calculated the proportion of the population sampled. Our second hypothesis was that both the methods would be biased towards the capture of smaller organisms. To test this hypothesis, we compared the body size of individuals that were captured and not captured to the population mean, for each method.

\section{Methods}

Test organisms

The freshwater amphipod G. pulex was selected as the test organism for laboratory experiments, because it is abundant in the surface and subsurface sediments of 
many temperate-zone streams (Crane, 1994; Wood et al., 2010; Stubbington et al., 2011a, b), and populations can easily be maintained in laboratory conditions (Vadher et al., 2015; Vander Vorste et al., 2016b). G. pulex were collected from a small lowland stream $(52.78 \mathrm{~N},-1.39 \mathrm{E})$ where it was the only amphipod present and occurred at densities of $>100$ individuals $\mathrm{m}^{-2}$. Collected organisms were kept at an ambient temperature of $13^{\circ} \mathrm{C}$ in tanks containing 81 continuously aerated, dechlorinated tap water. Individuals of all sizes (and therefore both sexes) were retained to allow subsequent body size analysis. Tanks were subject to an 8:16 h light:dark regime. G. pulex were fed ad libitum on pre-conditioned native leaf litter dominated by beech (Fagus sylvatica) and ash (Fraxinus excelsior).

Pump-sampling equipment

Bou-Rouch sampling equipment was purchased from Uwitec (Mondsee, Austria) and used unmodified. The Bou-Rouch standpipe was $125 \mathrm{~cm}$ long with a 20-mm internal diameter and a perforated section that spanned $15 \mathrm{~cm}$ and comprised 355 -mm diameter holes in 7 columns of 5 holes. Vacuum-pump sampling pipes each comprised a 25-cm length of PVC pipe with an internal diameter of $19 \mathrm{~mm}$, with samples extracted using a manual bilge pump.

\section{Sediment treatments}

Five sediment treatments were used to represent a range of possible field conditions: (i) $100 \%$ coarse (16-32 mm) gravel, (ii) $100 \%$ medium $(8-16 \mathrm{~mm})$ gravel, (iii) coarse gravel with $10 \%$ interstitial volume filled with fine (4-8 mm) gravel, (iv) coarse gravel with $30 \%$ interstitial volume filled with fine gravel, and (v) 100\% fine gravel. For treatments (iii) and (iv), the mean interstitial volume in the coarse gravel treatment was determined as 4.21 per mesocosm, and the quantity of fine gravel required to displace 420 or $1260 \mathrm{ml}$ of water (i.e. $10 \%$ or $30 \%$ of 4.2 1) was subsequently determined, and these sediment volumes were used in the respective treatments.

\section{Mesocosm design}

Each experimental mesocosm comprised an opentopped cylinder $(46 \mathrm{~cm}$ height $\times 28 \mathrm{~cm}$ diameter $)$ constructed from 1-mm wire mesh and lined with $<1$ mm mesh muslin cloth (Fig. 1). Mesocosms were placed on a base of 16-32 $\mathrm{mm}$ gravel within a rigid polyethylene cylindrical container $(74 \mathrm{~cm}$ height $\times$ $36 \mathrm{~cm}$ base internal diameter; Fig. 1).

For each treatment, sediment particles were added to the mesocosm to a depth of $15 \mathrm{~cm}$ (i.e. to the top of the perforated section of a Bou-Rouch standpipe), to ensure that all test organisms were within the sampled sediments during sample collection. One beech leaf and one ash leaf were broken into small $(<5 \mathrm{~mm}$ diameter) pieces and evenly distributed across the sediment at 5 and $10 \mathrm{~cm}$ above the mesocosm base, to act as a food resource prior to the start of an experimental trial. Dechlorinated tap water was added to the container to a depth approximately $4 \mathrm{~mm}$ below the substrate surface, to ensure all G. pulex were within subsurface interstices during sample collection. Mesocosms were kept at an air temperature of $13^{\circ} \mathrm{C}$.

In Bou-Rouch experiments, prior to sediment addition, the standpipe tip was pushed through a hole in the mesocosm base into the gravel below; the muslin cloth then formed a tight seal around the standpipe and gravel covered the lower, non-perforated part of the standpipe. In vacuum-pump experiments, a sampling pipe was placed onto the end of a stainless steel T-bar, which was then inserted into the sediment-filled mesocosm to a depth of $7.5 \mathrm{~cm}$ (i.e. the mid-point of the depth range for Bou-Rouch sampling; Fig. 1).

Water temperature was maintained at $13.2 \pm$ $0.05^{\circ} \mathrm{C}, \mathrm{pH}$ at $7.6 \pm 0.07$, conductivity at $549 \pm 43$ $\mu \mathrm{S} \mathrm{cm}^{-1}$, and dissolved oxygen concentrations at $6.0 \pm 0.2 \mathrm{mg}^{-1}$ during experiments. These parameters were measured using standard instrumentation (Hanna Instruments, Leighton Buzzard, UK) in the second 2-1 aliquot of water extracted with vacuumpump samples (see below).

\section{Experimental procedure}

Four replicate samples were collected for each sediment treatment and for both methods, totalling 40 experimental trials. Approximately $16 \mathrm{~h}$ before an experimental trial, $50 \mathrm{G}$. pulex were transferred from the tanks to a mesocosm in $75 \mathrm{ml}$ of water, increasing the water depth by $\leq 1 \mathrm{~mm}$ to approximately $3 \mathrm{~mm}$ below the substrate surface. Individuals were introduced evenly across the substrate surface and left to 
Fig. 1 Experimental mesocosms used to collect pump samples of a Gammarus pulex population from subsurface sediments. Either a Bou-Rouch standpipe or a vacuumpump sampling pipe was inserted into the "pipe insertion point" prior to sample extraction. Not to scale

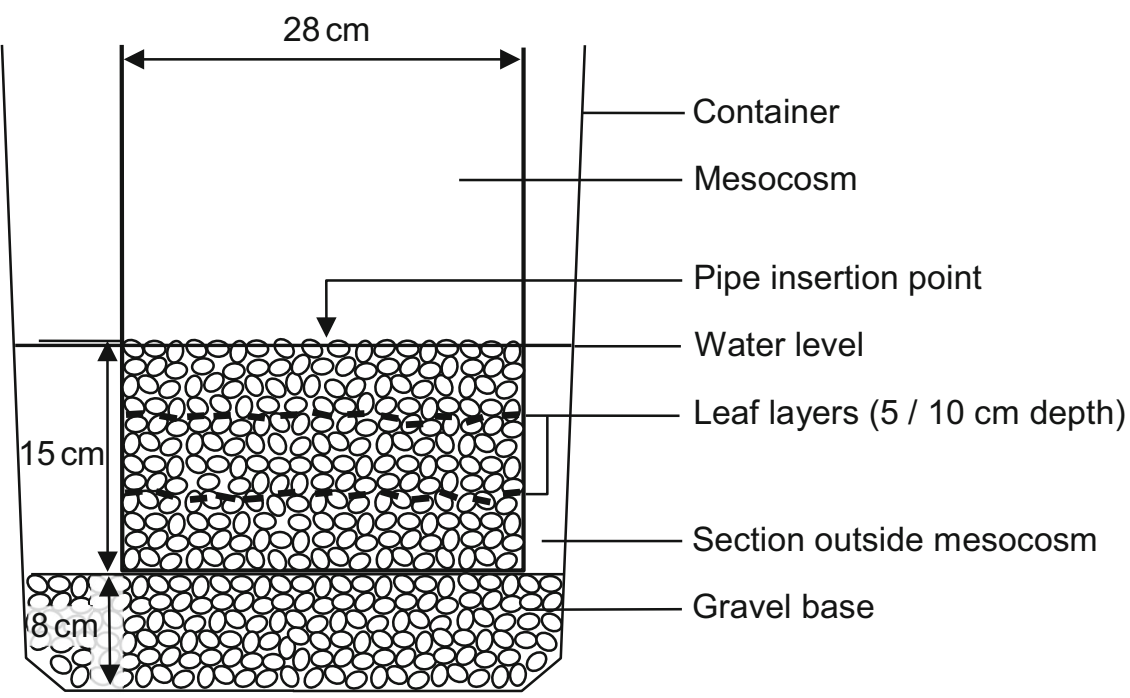

move into submerged interstices. During the $16 \mathrm{~h}$ pretrial period, constant artificial light was used to encourage G. pulex (which are negatively phototactic; Bakker et al., 1997) to move into deeper sediments, rather than remaining near the substrate surface. Positioning of leaf layers at depths of 5 and $10 \mathrm{~cm}$ was also intended to encourage vertical migration of G. pulex into subsurface sediments.

A 6-1 sample volume was collected, as this is typical of hyporheic macroinvertebrate studies and hence facilitated comparison with previous work (Boulton et al., 1992; Scarsbrook \& Halliday, 2002; Boulton et al., 2004b; Wood et al., 2010; Stubbington et al., 2011a, b; Datry, 2012). At the start of Bou-Rouch sample collection, a 500-ml volume of dechlorinated tap water was used to prime the apparatus. The BouRouch pump was then manually operated to extract a 6.5-1 sample (a 6-1 invertebrate sample and $500 \mathrm{ml}$ priming water). To collect a vacuum-pump sample, a length of hose was inserted into the pipe and the bilge pump operated manually to extract three 2-1 aliquots (i.e. one 6-1 sample) from the pipe base. During sample collection, water was continuously added to the space external to the mesocosm (Fig. 1) to prevent a decline in the water depth. Pumps were operated at the fastest rate possible, determined as $0.43 \mathrm{l} \mathrm{s}^{-1}$ for the BouRouch pump $(n=6)$ and $0.17 \mathrm{l} \mathrm{s}^{-1}$ for the vacuum pump $(n=6)$. Although standardization of pumping rate is desirable within each method (Hunt \& Stanley, 2000), using a consistent rate between methods would have been impractical and would have resulted in ineffective operation of the Bou-Rouch pump.

Extracted samples were passed through a 1-mm mesh net to retain all captured amphipods. Mesocosms were then deconstructed and the contents rinsed through sieves to locate G. pulex that had not been sampled. Sampled and non-sampled amphipods were preserved in $70 \%$ industrial methylated spirits and stored at $13^{\circ} \mathrm{C}$ for later analysis.

Measurement of test organisms

The body size of all sampled and non-sampled $G$. pulex was measured, with the exception of individuals that were damaged during passage through the BouRouch apparatus or during mesocosm deconstruction (7\% of sampled individuals and $27 \%$ of non-sampled individuals). Body size was measured from the base of antenna 1 to the posterior margin of the final urosome segment, using Motic Image Plus software version 2.0 (Motic, Hong Kong).

\section{Data analysis}

Analyses were conducted in IBM SPSS Statistics Version 22 (IBM Corporation, New York, USA), with proportional data $\arcsin (\sqrt{ } X)$-transformed prior to analysis. Results are stated as mean \pm 1 standard error (SE). 
Estimation of population size

To test our first hypothesis, we examined differences in the proportion of the G. pulex population captured by the two methods in the five sediment treatments using a two-way ANOVA test, with method (BouRouch; vacuum pump) and sediment treatment (coarse; medium; $10 \%$ fine; $30 \%$ fine; fine) as fixed factors, and with the proportion captured as the dependent variable. A significant interaction between sediment treatment and method was found (see below) and this initial test was therefore supplemented by: one-way ANOVA tests, to determine differences in capture rates between methods for each individual sediment treatment; one-way ANOVA tests with post hoc Tukey's tests, to examine differences in capture rates between treatments for each individual method.

\section{Characterization of body size}

To test our second hypothesis, we examined differences in the body size of G. pulex sampled by the two methods and differences in the size of sampled and non-sampled individuals. This was achieved using a three-way ANOVA test with method, sediment treatment, and sampling outcome (sampled; not sampled) as fixed factors and with mean organism size (calculated from 4 replicates) as the dependent variable. Where significant interactions between fixed factors were identified, one-way ANOVA tests were conducted to test for differences between groups. A separate one-sample $t$ test was used to compare the body size of organisms sampled by each method with the known population mean.

\section{Results}

Estimation of population size

Considering all 40 experimental trials (i.e. both methods and all sediment treatments), $18.9 \pm 3.1 \%$ (range $0-48 \%$ ) of the 50-individual G. pulex population was retrieved from mesocosms. Considering all sediment treatments, a higher proportion of the population was captured by Bou-Rouch sampling than by vacuum-pump sampling (Table 1 ; Fig. 2). The interaction between method and sediment treatment was significant (two-way ANOVA, $P=0.005$ ): the
Bou-Rouch method captured more individuals than vacuum-pump sampling in medium, fine, and mixed sediment treatments but there was no difference in the proportion of individuals captured in the $100 \%$ coarse gravel treatment (Table 1; Fig. 2). The comparable proportion captured by the two methods in the coarse gravel treatment reflected the lowest proportion sampled by the Bou-Rouch method (Tukey's test, $P=0.040$ compared to coarse gravel with $30 \%$ fine gravel) and the highest proportion collected by the vacuum pump $(P=0.028$ compared to $100 \%$ fine gravel; Table 1; Fig. 2).

\section{Characterization of body size}

The mean body size of $G$. pulex individuals was $9.0 \pm 0.2 \mathrm{~mm}$ (range 2.0-18.4 mm, $n=1623$ ). Considering both methods, captured individuals were smaller $(8.3 \pm 0.3 \mathrm{~mm}, n=378)$ than those which were not captured $(9.5 \pm 0.2 \mathrm{~mm}, n=1252$; threeway ANOVA, $P=0.001)$. The interaction between sampling method and sampling outcome was significant (three-way ANOVA, $P=0.015$ ): sampled $G$. pulex were smaller than non-sampled individuals in vacuum-pump samples (one-way ANOVA, $P<$ 0.001 ), but there was no significant difference in the body size of organisms sampled and not sampled using the Bou-Rouch method $(P=0.291$; Fig. 3$)$. At $9.2 \pm 0.4 \mathrm{~mm}(n=324)$, the size of $G$. pulex in Bou-Rouch samples was comparable to the population mean (one-sample $t$ test, $P=0.542$ ); at $7.0 \pm 0.2 \mathrm{~mm}$, individuals in vacuum-pump samples were on average $1.9 \mathrm{~mm}$ smaller than the population mean $(P=0.003$; Fig. 3$)$. There was no difference in the number of individuals sampled in the five sediment treatments (three-way ANOVA, $P=0.169$ ) or any significant interaction between treatment and either other fixed factor (method; sampling outcome, $P \geq 0.310$ ).

\section{Discussion}

Bou-Rouch sampling captured more invertebrates than vacuum-pump sampling

In four of five sediment treatments, our first hypothesis was supported: Bou-Rouch sampling captured a higher proportion of an invertebrate population than 
Table 1 Mean \pm 1 SE percentage of a known-size population of Gammarus pulex captured by Bou-Rouch and vacuum-pump sampling techniques in five sediment treatments

\begin{tabular}{|c|c|c|c|c|}
\hline \multirow[t]{2}{*}{ Sediment treatments } & \multicolumn{2}{|c|}{$\%$ Captured by method } & \multirow[t]{2}{*}{$F$} & \multirow[t]{2}{*}{$P$} \\
\hline & Bou-Rouch & Vacuum-pump & & \\
\hline All & $32.6 \pm 4.5$ & $5.1 \pm 1.1$ & 69.84 & 0.001 \\
\hline $100 \%$ Coarse gravel & $14.0 \pm 3.2$ & $12.0 \pm 2.6$ & 0.66 & 0.806 \\
\hline $100 \%$ Medium gravel & $41.5 \pm 10.2$ & $4.5 \pm 1.0$ & 20.06 & 0.004 \\
\hline Coarse gravel $+10 \%$ volume fine gravel & $26.0 \pm 8.6$ & $2.5 \pm 1.5$ & 11.18 & 0.016 \\
\hline Coarse gravel $+30 \%$ volume fine gravel & $52.0 \pm 12.6$ & $3.5 \pm 2.2$ & 19.08 & 0.005 \\
\hline $100 \%$ Fine gravel & $34.5 \pm 6.4$ & $1.5 \pm 1.5$ & 34.70 & 0.001 \\
\hline
\end{tabular}

Sediment particle sizes are stated in the text. Significance was determined using two-way (all treatments) and one-way (individual treatments) ANOVA tests

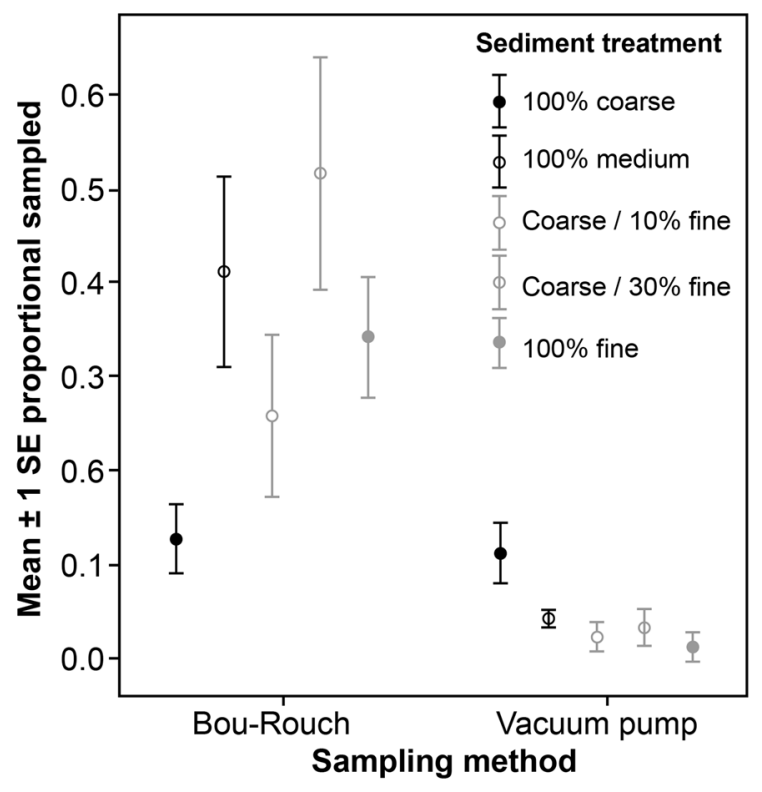

Fig. 2 Mean \pm 1 SE proportion of a 50-individual Gammarus pulex population captured from subsurface sediments using two pump-sampling methods (Bou-Rouch; vacuum pump) in five sediment treatments (100\% coarse gravel, $100 \%$ medium gravel, coarse gravel with $10 \%$ interstitial volume filled with fine gravel, coarse gravel with $30 \%$ interstitial volume filled with fine gravel, $100 \%$ fine gravel); $n=4$ for each treatment

vacuum-pump sampling. These results are consistent with our previous field-based comparison of these pump-sampling methods, in which we recorded higher abundance estimates in Bou-Rouch samples for all common taxa in six streams across two bioregions (Stubbington et al., 2016). We suggest that these higher abundance estimates reflect the stronger

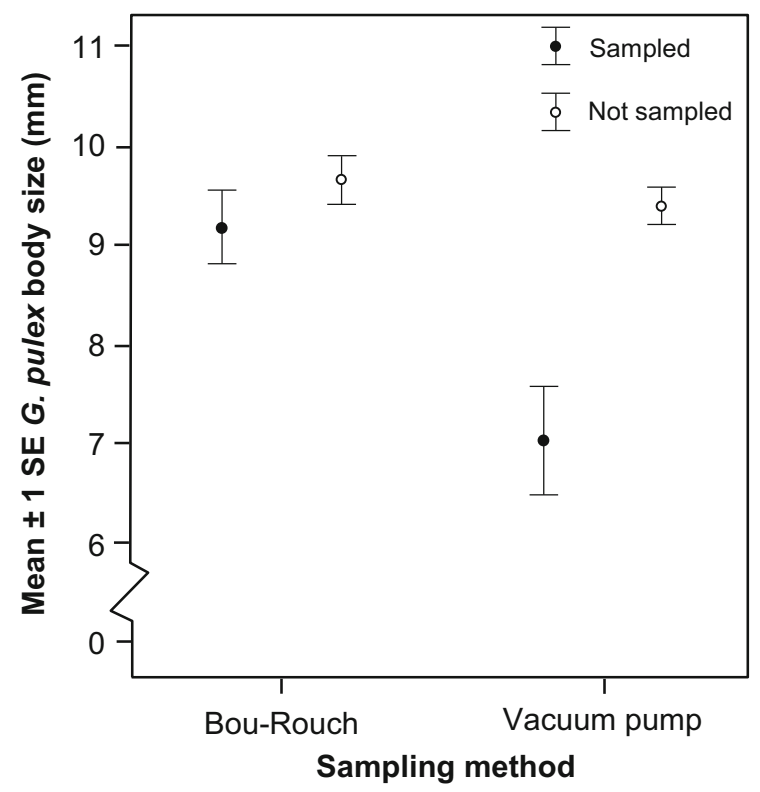

Fig. 3 Mean \pm 1 SE body size (mm) of Gammarus pulex individuals captured and not captured by Bou-Rouch and vacuum-pump sampling methods. Body size was measured from the base of antenna 1 to the posterior margin of the final urosome segment; $n=324$ for Bou-Rouch, sampled, $n=526$ for Bou-Rouch, not sampled, $n=54$ for vacuum pump, sampled, and $n=726$ for vacuum pump, not sampled

suction forces generated by the Bou-Rouch pump, which allow more rapid sample extraction than the manual bilge pump typically used in vacuum-pump sampling (Boulton et al., 1992). Similarly, Hunt \& Stanley (2000) compared different modes of operating the Bou-Rouch pump and found that faster pumping rates increased population density estimates. 
Pump sampling exposes invertebrates in sampled sediments to a sudden increase in flow velocity. When such increases occur naturally, for example at the onset of a spate, macroinvertebrates including $G$. pulex may respond by moving through interstitial pathways into sediments with lower velocities (DoleOlivier et al., 1997). As such, stronger swimming ability may help amphipods and other invertebrates to avoid both displacement by increasing flow velocities during floods and entrainment in faster-moving water during pump sampling (Boulton et al., 2004a).

The relationship between sample extraction rates and population densities observed by Hunt \& Stanley (2000), and the results of this study, might suggest that pump-sampling techniques capture invertebrates most effectively in coarse sediments, in which wide interstitial pathways facilitate rapid sample extraction. However, in our $100 \%$ coarse gravel treatment, the proportion of the population captured was comparable between techniques, being particularly low in BouRouch samples and particularly high in vacuum-pump samples. The higher capture rates achieved by the vacuum pump in coarse gravel may reflect reductions in both interstitial pathway blockages by finer sediment particles and in organisms trapped between particles. In contrast, the reduced effectiveness of the Bou-Rouch technique in coarse gravel may be due to the greater hydraulic conductivity (Datry et al., 2015) and therefore reduced suction forces associated with larger interstices: in finer and more heterogeneous sediment treatments, the lower interstitial pore volume would have increased suction forces.

Pump sampling underestimated population densities

The field study of Stubbington et al. (2016) also demonstrated that Bou-Rouch sampling provides higher invertebrate abundance estimates than vacuum-pump sampling. However, although such field studies allow comparisons, for example, between bioregions and/or streams, they do not indicate the proportion of a population that is sampled. In our controlled mesocosm study, both pump-sampling techniques consistently captured $<50 \%$ of the amphipod population present. In a related study, DoleOlivier et al. (2014) operated a Bou-Rouch pump within a quantitative Hess sampler to determine the proportion of an invertebrate assemblage collected by the pump in a stream with cobble to coarse-sand-sized sediments. On average, Dole-Olivier et al. (2014) found that the Bou-Rouch pump collected $14.5 \%$ of the invertebrates present in Hess samples, lower than our 33\% mean Bou-Rouch capture rate for G. pulex, which may reflect differences in environmental parameters such as substrate composition. This experimental identification of low capture rates informs interpretation of biotic data collected using pumpsampling techniques. However, further research is needed to investigate capture rates for other taxa and to compare rates for a range of environmental conditions and invertebrate traits.

Density estimates decrease as sample volume increases, and therefore, to maximize the accuracy of hyporheic population size estimates obtained using a pump-sampling technique, a small sample volume (Hunt \& Stanley, 2000; Boulton et al., 2003) and sufficient replicates (Kibichii et al., 2009) should be collected. Conversely, where a study aims to characterize taxa richness, a larger sample volume may be required; accordingly, several previous studies have collected 10-1 pump samples (e.g. Malard et al., 2003; Boon et al., 2016). However, where accurate population density estimates are required, we do not recommend pump-sampling methods as appropriate. Instead, fully quantitative methods such as freezecoring with electro-positioning (Bretschko \& Klemens, 1986; Marchant, 1995) or colonization devices (Gibbins et al., 2016, but see Fraser \& Williams, 1997) should be considered.

Bou-Rouch but not vacuum-pump sampling accurately characterized body size

Our second hypothesis, that both methods would be more likely to capture smaller individuals, was based on the size bias associated with pump sampling (Fraser \& Williams, 1997; Mauclaire et al., 1998; Boulton et al., 2004a) being common to both techniques. Our results lead us to reject this hypothesis: although organisms captured in vacuum-pump samples were smaller than the population mean, the body size of amphipods in Bou-Rouch samples reflected the population present. The smaller body size of amphipods in vacuum-pump samples indicates that larger individuals could escape the weaker suction forces exerted by this technique, while individuals from across the size range present were drawn into the Bou-Rouch 
standpipe. Larger organisms' greater ability to resist capture by weaker suction forces may reflect enhanced friction forces between organisms and sediment particles (Fraser \& Williams, 1997; Scarsbrook \& Halliday, 2002); the stronger swimming ability of adults compared to juveniles (Conlan, 1994); and/or the greater activity of larger males (Peeters et al., 2009), all of which facilitate the innate positive rheotaxis of G. pulex (Hughes, 1970).

The vacuum-pump sampling bias towards the capture of smaller individuals, which we observed across sediment treatments, is consistent with previous field studies. In particular, Scarsbrook \& Halliday (2002) compared vacuum-pump sampling to colonization pots and freeze-coring and found this method to overestimate the relative abundance of Ostracoda and Copepoda (i.e. very small taxa) compared to other methods and to underestimate the occurrence of mayfly nymphs, stonefly nymphs, and caddisfly larvae (i.e. larger organisms). This size bias, and its implicit exclusion of both individual organisms and potentially whole taxa, may limit the accuracy with which vacuum-pump sampling characterizes invertebrate community composition. Alongside the lower capture rates of this method, the size bias quantified by this experimental study may partly explain the finding of Stubbington et al. (2016) that vacuum-pump sampling characterizes differences in community composition among streams less effectively than Bou-Rouch sampling.

The absence of a size bias in Bou-Rouch experiments partly contrasts with Fraser \& Williams (1997), who noted that at a depth of $20 \mathrm{~cm}$ (but not 40 or $60 \mathrm{~cm}$ ) chironomid (Diptera) larvae collected from one riffle using a Bou-Rouch pump analogue were smaller than those in quantitative freeze-coring and standpipe corer samples. Although direct comparison of our results with Fraser \& Williams (1997) is limited by contrasting methodological and taxonomic details, the absence of a size bias in Bou-Rouch samples in our study could reflect stronger suction forces generated in experiments, which were conducted in sediments with minimal organic matrix and therefore unclogged interstitial pathways. In contrast, the size bias we observed in vacuum-pump samples supports Fraser \& Williams' (1997) suggestion that larger organisms have a greater capacity to resist entrainment by moving water. However, the presence of a size bias in vacuum-pump but not Bou-Rouch samples in our study suggests that the size bias is overcome as suction forces increase.

Given that the body size of G. pulex in Bou-Rouch samples reflected the population mean, we suggest that this method is appropriate for studies seeking to characterize both absolute and relative organism size, for example in relation to environmental characteristics or population densities (Schmid et al., 2000). However, further research is required to determine whether the lack of size bias we observed for the amphipod G. pulex is representative of other taxa. In addition, despite organism size in Bou-Rouch samples reflecting the population mean across sediment treatments with differing interstitial fine gravel content, comparison of these results (i.e. the lack of size bias in Bou-Rouch samples) with the size bias observed both in our vacuum-pump samples and by Fraser \& Williams (1997) highlights that environmental characteristics and taxon traits interact to determine individual responses to sample extraction (Stubbington, 2012). In particular, Scarsbrook \& Halliday (2002) caution that comparisons of organism size should only be conducted among sites with similar hydraulic conductivity, due to its influence on the tenacity of invertebrates exposed to suction forces.

Characterization of hyporheic invertebrate communities: future challenges

The subsurface sediments of the hyporheic zone provide a habitat for a diverse invertebrate fauna. In addition, the role of these sediments as a refuge during instream disturbances (Dole-Olivier, 2011; Stubbington, 2012) may mean that they become an increasingly important ecosystem component where extreme hydrological conditions become more common in a changing climate (Ledger \& Milner, 2015). Understanding the limitations of current sampling methods is therefore important to inform interpretation of field sampling data. Pumping methods are widely used and their use may further increase if hyporheic communities become new biomonitors of ecosystem health (Leigh et al., 2013; Boulton, 2014) under the requirements of international legislation. Our study contributes to recent work seeking to inform both interpretation of research data and development of biomonitoring programmes by quantifying the limitations of such hyporheic invertebrate sampling methods (Dole-Olivier et al., 2014; Stubbington et al., 2016). 
We demonstrated that vacuum-pump sampling collects a particularly small proportion (mean $5.1 \pm 1.1 \%$ ) of the population present and that the individuals captured have a body size less than the population mean. This low proportion and size bias may both restrict vacuum-pump sampling's characterization of community composition, with larger, more adherent, more robust, and more active taxa and individuals potentially less likely to be sampled. Such limitations may prevent standard vacuum-pump sampling from distinguishing between contrasting invertebrate communities (e.g. Scarsbrook \& Halliday, 2002; Stubbington et al., 2016), although the method has previously proved capable of identifying both spatial and temporal changes in community composition (Boulton et al., 1992; Stubbington et al., 2011a, b). Adaptation of standard vacuum-pump sampling, for example using a higher flow-rate manual bilge pump or an electric pump (Tanaka et al., 2014), may improve the method's performance by increasing sample extraction rates. In contrast, although BouRouch pumping also extracted a limited proportion of the population present (mean $32.6 \pm 4.5 \%$; also see Dole-Olivier et al., 2014), this technique sampled organisms from the entire population size distribution.

A recognized limitation of all pump-sampling methods is the unknown position of the sampled sediments (Roy \& Danielescu, 2014). Our study describes a simple mesocosm set-up that could be adapted to address such research gaps. We recommend that further experimental work be conducted to determine where sampled invertebrates originate from, in terms of both their vertical distribution and their proximity to the sampling pipe. In addition, our study examined a single taxon, and further research is required to examine the effectiveness of pump sampling for capturing a wide range of taxa, including both macroinvertebrates and meiofauna.

Acknowledgments Practical work conducted by JPH and Ben Stephens was funded by Nottingham Trent University bursaries. The authors thank Jackie Greef and Mick Shaw for providing technical assistance in the laboratory and field. Many thanks to Mark Szegner for technical assistance with figure production. We also acknowledge the contributions of two anonymous reviewers, whose comprehensive and insightful comments greatly improved an earlier version of this manuscript.

Open Access This article is distributed under the terms of the Creative Commons Attribution 4.0 International License (http:// creativecommons.org/licenses/by/4.0/), which permits unrestricted use, distribution, and reproduction in any medium, provided you give appropriate credit to the original author(s) and the source, provide a link to the Creative Commons license, and indicate if changes were made.

\section{References}

Bakker, T. C. M., D. Mazzi \& S. Zala, 1997. Parasite-induced changes in behavior and color make Gammarus pulex more prone to fish predation. Ecology 78: 1098-1104.

Boon, P. J., N. Willby, D. Gilvear \& D. Pryce, 2016. The regional hyporheic fauna of gravel-bed rivers and environmental controls on its distribution. Fundamental and Applied Limnology 187: 223-239.

Bou, C. \& R. Rouch, 1967. Un nouveau champ de recherches sur la faune aquatique souterraine. Comptes Rendus de l'Académie des Sciences de Paris 265: 369-370.

Boulton, A. J., 2000. The subsurface macrofauna. In Jones, J. B. \& P. J. Mulholland (eds), Streams and Ground Waters. Academic, San Diego: 337-362.

Boulton, A. J., 2014. Conservation of ephemeral streams and their ecosystem services: What are we missing? Aquatic Conservation 24: 733-738.

Boulton, A. J., H. M. Valett \& S. G. Fisher, 1992. Spatial distribution and taxonomic composition of the hyporheos of several Sonoran Desert streams. Archiv für Hydrobiologie 125: 37-61.

Boulton, A. J., M. J. Dole-Olivier \& P. Marmonier, 2003. Optimizing a sampling strategy for assessing hyporheic invertebrate biodiversity using the Bou-Rouch method: within-site replication and sample volume. Archiv für Hydrobiologie 156: 431-456.

Boulton, A. J., M. J. Dole-Olivier \& P. Marmonier, 2004a. Effects of sample volume and taxonomic resolution on assessment of hyporheic assemblage composition sampled using a Bou-Rouch pump. Archiv für Hydrobiologie 159: 327-355.

Boulton, A. J., M. Harvey \& H. Proctor, 2004b. Of spates and species: responses by interstitial water mites to simulated spates in a subtropical Australian river. Applied and Experimental Acarology 34: 149-169.

Boulton, A. J., G. D. Fenwick, P. J. Hancock \& M. S. Harvey, 2008. Biodiversity, functional roles and ecosystem services of groundwater invertebrates. Invertebrate Systematics 22: 103-116.

Bretschko, G. \& W. E. Klemens, 1986. Quantitative methods and aspects in the study of the interstitial fauna of running waters. Stygologia 2: 297-316.

Conlan, K., 1994. Amphipod crustaceans and environmental disturbance: a review. Journal of Natural History 28: 519-554.

Crane, M., 1994. Population characteristics of Gammarus pulex (L.) from five English streams. Hydrobiologia 281: 91-100.

Datry, T., 2012. Benthic and hyporheic invertebrate assemblages along a flow intermittence gradient: effects of duration of dry events. Freshwater Biology 57: 563-574. 
Datry, T., N. Lamouroux, G. Thivin, S. Descloux \& J. Baudoin, 2015. Estimation of sediment hydraulic conductivity in river reaches and its potential use to evaluate streambed clogging. River Research and Applications 31: 880-891.

Davy-Bowker, J., W. Sweeting, N. Wright, R. T. Clarke \& S. Arnott, 2006. The distribution of benthic and hyporheic macroinvertebrates from the heads and tails of riffles. Hydrobiologia 563: 109-123.

Dole-Olivier, M. J., 2011. The hyporheic refuge hypothesis reconsidered: a review of hydrological aspects. Marine and Freshwater Research 62: 1281-1302.

Dole-Olivier, M. J. \& P. Marmonier, 1992. Effects of spates on the vertical distribution of the interstitial community. Hydrobiologia 230: 49-61.

Dole-Olivier, M. J., P. Marmonier \& J. L. Beffy, 1997. Response of invertebrates to lotic disturbance: Is the hyporheic zone a patchy refugium? Freshwater Biology 37: 257-276.

Dole-Olivier, M. J., F. Castellarini, N. Coineau, D. M. P. Galassi, P. Martin, N. Mori, A. Valdecasas \& J. Gibert, 2009. Towards an optimal sampling strategy to assess groundwater biodiversity: comparison across six European regions. Freshwater Biology 54: 777-796.

Dole-Olivier, M. J., C. Maazouzi, B. Cellot, F. Fiers, D. M. P. Galassi, C. Claret, D. Martin, S. Merigoux \& P. Marmonier, 2014. Assessing invertebrate assemblages in the subsurface zone of stream sediments (0-15 cm deep) using a hyporheic sampler. Water Resources Research 50: 453-465.

European Commission, 2000. Directive 2000/60/EC of the European Parliament and of the Council establishing a framework for Community action in the field of water policy. Official Journal of the European Union 43: 1-72.

Fraser, B. G. \& D. D. Williams, 1997. Accuracy and precision in sampling hyporheic fauna. Canadian Journal of Fisheries and Aquatic Sciences 54: 1135-1141.

Gibbins, C., J. Grant, I. Malcolm \& C. Soulsby, 2016. Influence of groundwater chemistry on hyporheic invertebrate assemblages is revealed by fine-scale sampling. Fundamental and Applied Limnology 187: 207-221.

Hughes, D., 1970. Some factors affecting drift and upstream movements of Gammarus pulex. Ecology 51: 301-305.

Hunt, G. W. \& E. H. Stanley, 2000. An evaluation of alternative procedures using the Bou-Rouch method for sampling hyporheic invertebrates. Canadian Journal of Fisheries and Aquatic Sciences 57: 1545-1550.

ISO, 2015. Water Quality - Guidance on Methods for Sampling Invertebrates in the Hyporheic Zone of Rivers. ISO 16772, International Organization for Standardization.

Jones, I., I. Growns, A. Arnold, S. McCall \& M. Bowes, 2015. The effects of increased flow and fine sediment on hyporheic invertebrates and nutrients in stream mesocosms. Freshwater Biology 60: 813-826.

Kibichii, S., J. Baars \& M. Kelly-Quinn, 2009. Optimising sample volume and replicates using the Bou-Rouch method for the rapid assessment of hyporheic fauna. Marine and Freshwater Research 60: 83-96.

Ledger, M. E. \& A. M. Milner, 2015. Extreme events in running waters. Freshwater Biology 60: 2455-2460.

Leigh, C., R. Stubbington, F. Sheldon \& A. J. Boulton, 2013. Hyporheic invertebrates as bioindicators of ecological health in temporary rivers: a meta-analysis. Ecological Indicators 32: 62-73.
Malard, F., S. Plenet \& J. Gibert, 1996. The use of invertebrates in ground water monitoring: a rising research field. Groundwater Monitoring and Remediation 16: 103-113.

Malard, F., D. Galassi, M. Lafont, S. Doledec \& J. V. Ward, 2003. Longitudinal patterns of invertebrates in the hyporheic zone of a glacial river. Freshwater Biology 48: 1709-1725.

Marchant, R., 1995. Seasonal variation in the vertical distribution of hyporheic invertebrates in an Australian upland river. Archiv für Hydrobiologie 134: 441-457.

Marshall, M. C. \& R. O. Hall Jr., 2004. Hyporheic invertebrates affect $\mathrm{N}$ cycling and respiration in stream sediment microcosms. Journal of the North American Benthological Society 23: 416-428.

Mauclaire, L., P. Marmonier \& J. Gibert, 1998. Sampling water and sediment in interstitial habitats: a comparison of coring and pumping techniques. Archiv für Hydrobiologie 142: 111-123.

McGrath, K. E., E. T. H. M. Peeters, J. A. J. Beijer \& M. Scheffer, 2007. Habitat-mediated cannibalism and microhabitat restriction in the stream invertebrate Gammarus pulex. Hydrobiologia 589: 155-164.

Palmer, M. A., 1993. Experimentation in the hyporheic zone challenges and prospectus. Journal of the North American Benthological Society 12: 84-93.

Peeters, E. T., H. De Lange \& M. Lürling, 2009. Variation in the behavior of the amphipod Gammarus pulex. Human and Ecological Risk Assessment 15: 41-52.

Reyjol, Y., C. Argillier, W. Bonne, A. Borja, A. D. Buijse, A. C. Cardoso, M. Daufresne, M. Kernan, M. T. Ferreira, S. Poikane, N. Prat, A. L. Solheim, S. Stroffek, P. UsseglioPolatera, B. Villeneuve \& W. van de Bund, 2014. Assessing the ecological status in the context of the European Water Framework Directive: Where do we go now? Science of the Total Environment 497: 332-344.

Roy, J. W. \& S. Danielescu, 2014. Comment on “Assessing invertebrate assemblages in the subsurface zone of stream sediments (0-15 $\mathrm{cm}$ deep) using a hyporheic sampler" by Dole-Olivier et al. Water Resources Research 50: 9120-9123.

Scarsbrook, M. \& J. Halliday, 2002. Detecting patterns in hyporheic community structure: Does sampling method alter the story? New Zealand Journal of Marine and Freshwater Research 36: 443-453.

Schmid, P. E., M. Tokeshi \& J. M. Schmid-Araya, 2000. Relation between population density and body size in stream communities. Science 289: 1557-1560.

Stubbington, R., 2012. The hyporheic zone as an invertebrate refuge: a review of variability in space, time, taxa and behaviour. Marine and Freshwater Research 63: 293-311.

Stubbington, R., P. J. Wood \& I. Reid, 2011a. Spatial variability in the hyporheic zone refugium of temporary streams. Aquatic Sciences 73: 499-511.

Stubbington, R., P. J. Wood, I. Reid \& J. Gunn, 2011b. Benthic and hyporheic invertebrate community responses to seasonal flow recession in a groundwater-dominated stream. Ecohydrology 4: 500-511.

Stubbington, R., A. J. Boulton, S. Little \& P. J. Wood, 2015. Changes in invertebrate assemblage composition in benthic and hyporheic zones during a severe supraseasonal drought. Freshwater Science 34: 344-354. 
Stubbington, R., M. J. Dole-Olivier, D. M. P. Galassi \& P. J. Wood, 2016. Characterization of macroinvertebrate communities in the hyporheic zone of river ecosystems reflects the pump-sampling technique used. PLoS One 11(10): e0164372. doi: 10.1371/journal.pone.0164372.

Tanaka, A., T. Namba, K. Tanida \& Y. Takemon, 2014. Evaluation of a pump method for unbiased sampling of stream hyporheos. Hydrobiologia 730: 29-43.

Tomlinson, M., A. J. Boulton, P. J. Hancock \& P. G. Cook, 2007. Deliberate omission or unfortunate oversight: Should stygofaunal surveys be included in routine groundwater monitoring programs? Hydrogeology Journal 15: $1317-1320$

Vadher, A. N., R. Stubbington \& P. J. Wood, 2015. Fine sediment reduces vertical migrations of Gammarus pulex (Crustacea: Amphipoda) in response to surface water loss. Hydrobiologia 753: 61-71.
Vander Vorste, R., F. Malard \& T. Datry, 2016a. Is drift the primary process promoting the resilience of river invertebrate communities? A manipulative field experiment in an intermittent alluvial river. Freshwater Biology 61: 1276-1292.

Vander Vorste, R., F. Mermillod-Blondin, F. Hervant, R. Mons, M. Forcellini \& T. Datry, 2016b. Increased depth to the water table during river drying decreases the resilience of Gammarus pulex and alters ecosystem function. Ecohydrology. doi:10.1002/eco.1716.

Wood, P. J., A. J. Boulton, S. Little \& R. Stubbington, 2010. Is the hyporheic zone a refugium for aquatic macroinvertebrates during severe low flow conditions? Fundamental and Applied Limnology 176: 377-390. 